\title{
A systematic review of clinical and laboratory parameters of 3,000 COVID-19 cases
}

\author{
Harsh Goel, M.Tech ${ }^{1}$, Ishan Gupta, MBBS-Student ${ }^{2}$, Meenakshi Mourya, MBBS ${ }^{3}$, Sukhdeep Gill, MS, DABR ${ }^{4}$, \\ Anita Chopra, MD, DM¹, Amar Ranjan, MD¹, Goura Kishor Rath, MD5, Pranay Tanwar, MD

\begin{abstract}
${ }^{1}$ Laboratory Oncology Unit, Dr.B.R.A.-Institute Rotary Cancer Hospital, ${ }^{2}$ All India Institute of Medical Sciences, New Delhi; ${ }^{3}$ Department of Anaesthesia and Intensive Care, Vardhaman Mahavir Medical College \& Safdarjung Hospital, New Delhi, India, ${ }^{4}$ UPMC Hillman Cancer Center, Erie, PA, USA,
\end{abstract} \\ ${ }^{5}$ Department of Radiotherapy, Dr. B.R.A.- Institute Rotary Cancer Hospital, All India Institute of Medical Sciences, New Delhi, India
}

The coronavirus disease 2019 (COVID-19) has spread worldwide. It is still a pandemic and poses major health problem across the globe. In our review, clinical characteristics and laboratory parameters of COVID-19 patients were compiled systematically, with special reference to pregnant women in order to understand the disease course. An extensive literature search on various scientific databases for relevant manuscripts was conducted, which yielded 7 manuscripts for final analysis. The most common symptoms were fever (85\%), cough (70.63\%), chest tightness $(37.36 \%)$, expectoration (33.27\%), fatigue (32\%), dyspnea (31.95\%), and shortness of breath $(31.19 \%)$, while hemoptysis $(1.0 \%)$ was the least common. The associated comorbidities were hypertension $(21.6 \%)$ and diabetes $(10.0 \%)$. In terms of hematological parameters, lower total leukocyte counts were observed in $65 \%$ of cases and biochemical parameters, patients demonstrated elevated levels of albumin (53.72\%), lactate dehydrogenase (45.71\%), and natriuretic peptide (34.84\%); however, total bilirubin was elevated in only $8 \%$ of cases. In the acute inflammatory cytokine profile, C-reactive protein $(59.0 \%)$, tumor necrosis factor $(58.0 \%)$, erythrocyte sedimentation rate $(57.0 \%)$, interleukin-2 (IL$2,54.0 \%)$, and IL-6 (52.0\%) levels were increased, while prolactin levels $(6.5 \%)$ were minimally elevated. The recovery rate was approximately $41 \%$, and mortality was about $6.5 \%$. The study also concluded that the clinical symptoms of COVID-19 were similar among pregnant and non-pregnant women. There was no evidence of vertical transmission of COVID-19 infection. This review critically analyzed COVID-19 as a public health hazard in order to help policy makers, health care givers, and primary physicians to promote early diagnosis and prevention.

Keywords: COVID-19; SARS-CoV-2; 2019-nCoV; Coronavirus

\section{Introduction}

In early December 2019, the first case of pneumonia of unknown origin was identified in Wuhan. As of May 17, 2020, approximately 4,710,614 cases have been reported from over a hundred countries worldwide. More than 315,023 patients have died from severe infection with this novel virus, known as severe acute respiratory syndrome coronavirus 2 (SARS-CoV-2). This enveloped RNA, beta coronavirus was first identified by high-throughput sequencing. SARS-CoV-2 has a receptor-binding domain structure similar to that of SARS-COV, as shown with the help of homology modeling [1]. Zhou et al. [2] found that $96 \%$ of SARS-CoV-2 and bat coronaviruses are similar at the whole-genome level. The clinical disease course of this highly contagious illness, known coronavirus disease 2019 (COVID-19), has not yet been well described in the medical literature. During this ongoing pandemic, the center-specific clinical experience of COVID-19 is being shared by researchers worldwide. To date, there is no

Received: 2020.06.23. Revised: 2020.09.17. Accepted: 2021.01.11. Corresponding author: Pranay Tanwar, MD

Laboratory Oncology Unit, Dr.B.R.A.-Institute Rotary Cancer Hospital, All India Institute of Medical Sciences, Dr.B.R.A.Institute Rotary Cancer Hospital, Sri Aurobindo Marg, Ansari Nagar, New Delhi 110029, India

E-mail: pranaytanwar@gmail.com

https://orcid.org/0000-0002-2357-976X

Articles published in Obstet Gynecol Sci are open-access, distributed under the terms of the Creative Commons Attribution Non-Commercial License (http://creativecommons. org/licenses/by-nc/3.0/) which permits unrestricted non-commercial use, distribution, and reproduction in any medium, provided the original work is properly cited.

Copyright $\odot 2021$ Korean Society of Obstetrics and Gynecology 


\section{Obstetrics \& Gynecology Science}

Harsh Goel, et al. Review of 3,000 COVID-19 cases

effective vaccine or targeted therapy available. Therefore, supportive treatment alone is the mainstay of clinical management [3]. Subsequently, it's proven that the virus causing COVID-19 is similar to SARS-CoV and was designated as SARS-CoV-2 [4,5]. The COVID-19 outbreak was declared a "Public Health Emergency of International Concern" by the World Health Organization (WHO) on January 30, 2020 [6]. SARS-CoV-2 is capable of human-to-human transmission, and has spread worldwide by SARS-CoV-2-infected international travelers. In this study, we explored the clinical features and related laboratory parameters of more than 3,000 cases of COVID-19 and evaluated critical factors that may affect the disease course and prognosis. This review also focuses on the COVID-19 disease course and symptoms among pregnant women, with the intent to analyze the effect of COVID-19 on the progress of pregnancy and neonatal outcomes. We also evaluated the possibilities of vertical transmission of COVID-19 infection. This analysis is critically important and discusses COVID-19 as a public health hazard to "sensitize" the policymakers, health care givers, and primary physicians.

The human coronavirus is one of the primary pathogens of respiratory infections worldwide. The 2 highly pathogenic coronaviruses, SARS-COV and the Middle East respiratory syndrome-related coronavirus (MERS-CoV), cause severe respiratory syndrome in humans and have led to global epidemics in the past decades. Severe acute respiratory syndrome coronavirus 2 (SARS-CoV-2) is the coronavirus strain that causes a COVID-19 spread worldwide, posing a critical threat to global health. MERS-CoV is a species of coronavirus was first reported in Saudi Arabia in 2012, which is responsible for a viral respiratory disease $[7,8]$. In addition, 4 different human coronaviruses ( $\mathrm{HCOV}$ ) have been shown to induce mild upper respiratory disease, including $\mathrm{HCOV}-\mathrm{OC} 43, \mathrm{HCoV}$ 229E, HCoV-NL63, and HCoV-HKU1 [9]. Despite the high number of infections linked with COVID-19, it seems to have a lower death rate than either SARS-CoV or MERS- CoV [10-12].

The similarity of the phylogenetic profiles between SARSCoV-2 and SARS-CoV also lead to some overlapping clinical symptoms. Certain organs are more predisposed to COVID-19 infection when compared with SARS-CoV, MERS-CoV, and seasonal influenza infections. For example, seasonal influenza is more common in respiratory outpatient clinics and wards [13-15]. There are 6 coronavirus species currently known to cause infections in humans. SARS-CoV-2 is most closely linked to SARS-CoV as they share a similar recep- tor, that is, angiotensin-converting enzyme receptor-2. This evidence indicates that COVID-19 may partially mimic SARS [2]. The SARS-CoV mortality has been reported as more than $10 \%$, while MERS- CoV has been reported to be higher than $35 \%[16]$.

\section{Definition of COVID-19 cases}

The WHO recently updated and grouped the COVID-19 case definition into 3 categories: suspected, probable, and confirmed [17]. A suspected case is one that meets the clinical and epidemiological characteristics. Clinical features include acute onset of fever and cough with or without an acute onset of any three of the following, fever, cough, fatigue, headache, myalgia, sore throat, coryza, dyspnea, vomiting, and diarrhea. Epidemiological features include residing in a high transmission area, having traveled to a pandemic zone in the last 14 days, or working in a healthcare setting. A patient with severe acute respiratory illness (acute respiratory infection with a history of fever or measured fever of $\geq 38^{\circ} \mathrm{C}$; and cough; with onset within the last ten days; and requires hospitalization) is also considered as a suspected case. The criteria for a probable case are as follows: a) a patient who meets the clinical criteria mentioned above and is a contact of a probable or confirmed case, or epidemiologically linked to a cluster with at least one confirmed case; b) a suspected case with chest imaging showing findings suggestive of COVID-19 disease; c) a person with a recent onset of anosmia (loss of smell) or ageusia (loss of taste) in the absence of any other identified cause; d) death, not otherwise explained, in an adult with respiratory distress preceding death, and the person was a contact of a probable or confirmed case or epidemiologically linked to a cluster with at least 1 confirmed case. A confirmed case is a person with laboratory confirmation of COVID-19 infection, irrespective of clinical signs and symptoms.

\section{Methods}

\section{Literature search and selection}

We performed a systematic literature search of online databases, including PubMed, Google Scholar, Embase, and Web of Science, from December 2019 to May 2020, using the following search terms: COVID-19, 2019-nCoV, clinical characteristics, 2019 novel coronavirus, and SARS-CoV-2. All search 


\title{
Obstetrics \& Gynecology Science
}

\author{
Vol. 64, No. 2, 2021
}

results were evaluated according to the algorithm (Fig. 1), as mentioned. A total of 490 manuscripts were identified across various databases using the initial keyword search, as mentioned above. After eliminating 214 manuscripts as duplicates due to common MeSH terms, a total of 276 manuscripts were identified, which included 184 by abstracts and initial headings and 92 by detailed clinical parameters. Out of the 276 screened manuscripts, 126 were excluded after evaluating their scientific study design and clinical investigation. The remaining 150 were retrieved for full-text assessment. The studies that discussed clinical symptoms and laboratory parameters were included in the analysis. The studies that focused on basic research, small case studies, and case reports were screened further. After qualitative synthesis and application of our inclusion criteria, we further excluded 143 articles. Finally, we identified 7 manuscripts that were eligible for our systematic review.

\section{Study definitions, inclusion and exclusion criteria}

Studies were included if they satisfied the following criteria: identified target population; included cases with diagnosed COVID-19; and provided detailed accounts of clinical features and related laboratory parameters. Studies related to maternal and neonatal outcomes were compiled and evaluated separately using the same selection criteria. Studies mentioning follow-up and detailed assessment were only utilized for analysis. This review has a selection bias towards patients from the Asia Pacific region based on the geographic loca-

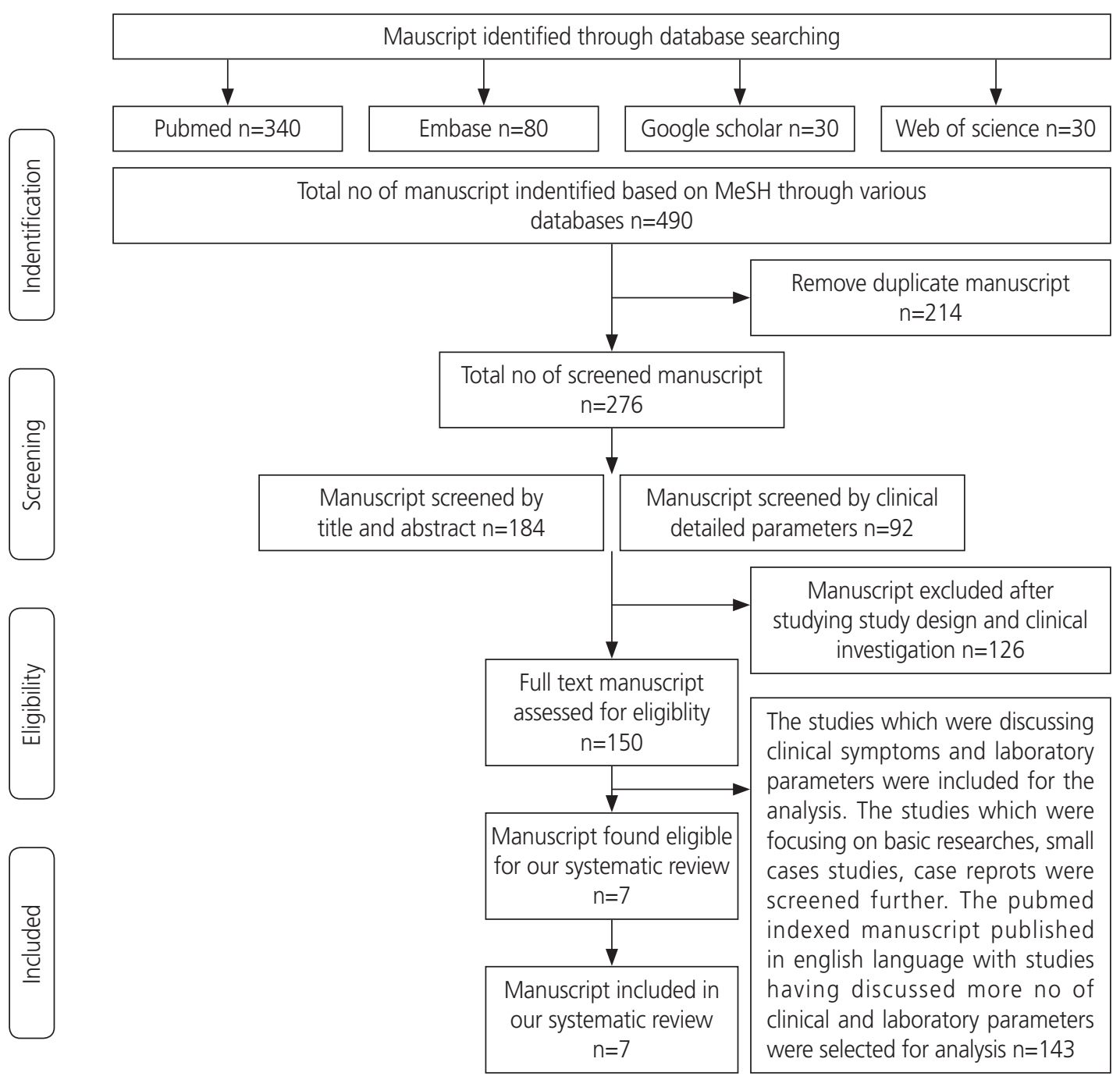

Fig. 1. Schematic representation of searching eligible manuscripts for analysis. 


\section{Obstetrics \& Gynecology Science}

Harsh Goel, et al. Review of 3,000 COVID-19 cases

tion of the initial COVID-19 outbreak. Of the seven studies, six were retrospective [4,18-22], and 1 by Li et al. [23] was an ambispective cohort study. All studies [24-28] describing COVID-19 symptoms in pregnant women were retrospective studies.

\section{Data extraction and quality assessment}

Both authors independently assessed the quality of the manuscript based on scientific methodology and case selection. Studies containing detailed clinical features and investigations were selected for analysis. Variables related to demographic characteristics, clinical features, associated comorbid conditions and related blood and biochemical investigations, treatment-related interventions, complication records, and duration of hospital stay were collected. None of the studies reported any variables; hence, the denominator was based on the availability of specific variable-related information in the manuscript. The entire process of data compilation was individually performed by two reviewers independently. Disagreements were resolved by mutual consensus.

\section{Bias risk assessment}

All manuscripts were selected based on strict inclusion criteria containing sufficient information of maximum possible variables. Scientific methodology as well as consecutive case selection were also ensured. However, stringent criteria for risk bias assessment related to the geographical region could not be applied, as selection bias favored the Asia-Pacific region. The identification of the bias coincided with the incidence of the initial COVID-19 epidemic in the Asia-Pacific region. Therefore, a majority of the recent pioneering studies evaluating clinical and laboratory parameters of COVID-19 have emerged from this region.

\section{Statistical analysis}

The relevant variables were analyzed from the individual manuscripts and were tabulated in Microsoft Excel sheets and expressed as event numbers, percentages, total events, and absolute frequency.

\section{Results}

\section{Observations of COVID-19 infection}

The overall analysis was categorized into two different groups: the general population (Tables 1-4), and pregnant women (Table 5).

\section{Age and sex distribution}

Seven studies from China, with a total of 3,231 subjects (1,786 male and 1,445 female), were analyzed. The median age was 53 years (range, 47-62 years) (Table 1), indicating that middle-aged adults were more prone to COVID-19. The minimum age of the patients was 20 years and the maximum was 91 years. The proportion of males (55.2\%) infected was higher than that of females (44.8\%). A history of smoking was not mentioned in any of the included studies; however, infection among current smokers was not as prominent as among non-smokers (83.80\%).

\section{Clinical symptoms}

A detailed account of the patients' clinical history and symptoms was collected from different studies and tabulated in order to determine the frequency list. Information on a total of 23 symptoms was collected from all the published studies. Although information on many symptoms was lacking, a detailed analysis was carried out from the available data. Fever (85.0\%) was the most common clinical manifestation, followed by cough (70.63\%) (Table 1). The data regarding fever and cough can be considered homogeneous as it was analyzed by all the authors. Other common symptoms included, in decreasing frequency, chest tightness (37.36\%), expectoration (33.27\%), fatigue (32.05\%), dyspnea (31.95\%), and shortness of breath (31.19\%). Minor symptoms included myalgia (15.48\%), diarrhea (11.39\%), headache (10.45\%), chills $(10.12 \%)$, sore throat $(9.40 \%)$, nausea, and vomiting (5.97\%). Abdominal pain and hemoptysis were considered rare symptoms as they reported very low frequencies (less than $5 \%$ ).

\section{Association among comorbid conditions}

Most infectious diseases affect immunocompromised individuals with associated comorbidities. The reports of associated comorbid conditions was also analyzed in detail (Table 2). However, there was little uniformity of data as associated morbidity-related cardinal information was not available in some of the analyzed studies. The final frequency percentage was calculated after reducing the number of total cases from the denominators if the data of a particular illness was not available in a specific study. The associated comorbid 


\section{Obstetrics \& Gynecology Science}

Vol. 64, No. 2, 2021

Table 1. Demographic details and list of clinical symptoms COVID-19

\begin{tabular}{|c|c|c|c|c|c|c|c|c|c|c|}
\hline Parameter & $\begin{array}{c}\text { Guan et } \\
\text { al. }[4] \\
(n=1,099)\end{array}$ & $\begin{array}{l}\text { Feng et } \\
\text { al. [18] } \\
(n=476)\end{array}$ & $\begin{array}{c}\text { Yu et } \\
\text { al. [19] } \\
(n=333)\end{array}$ & $\begin{array}{l}\text { Chen et } \\
\text { al. [20] } \\
(n=274)\end{array}$ & $\begin{array}{l}\text { Chen et } \\
\text { al. [21] } \\
(n=203)\end{array}$ & $\begin{array}{c}\text { Cai et } \\
\text { al. [22] } \\
(n=298)\end{array}$ & $\begin{array}{c}\text { Li et } \\
\text { al. [23] } \\
(n=548)\end{array}$ & $\begin{array}{l}\text { Events } \\
\text { number }\end{array}$ & Total & $\begin{array}{c}\text { Overall } \\
\text { percentage }\end{array}$ \\
\hline \multicolumn{11}{|l|}{ Age \& sex distribution } \\
\hline Age (yr) & $\begin{array}{c}47 \\
(35-58)\end{array}$ & $\begin{array}{c}53 \\
(40-64)\end{array}$ & $\begin{array}{c}50 \\
(35-63)\end{array}$ & $\begin{array}{c}62 \\
(44-70)\end{array}$ & $\begin{array}{c}54 \\
(20-91)\end{array}$ & $\begin{array}{c}47.5 \\
(33-61)\end{array}$ & $\begin{array}{c}60 \\
(48-69)\end{array}$ & - & - & - \\
\hline \multicolumn{11}{|l|}{ Sex } \\
\hline Female & $\begin{array}{c}459 \\
(41.8)\end{array}$ & $\begin{array}{c}205 \\
(43.1)\end{array}$ & $\begin{array}{c}161 \\
(48.3)\end{array}$ & $\begin{array}{c}103 \\
(38.0)\end{array}$ & $\begin{array}{c}95 \\
(46.8)\end{array}$ & $\begin{array}{c}153 \\
(51.34)\end{array}$ & $\begin{array}{c}269 \\
(49.1)\end{array}$ & 1,445 & 3,231 & 44.8 \\
\hline Male & $\begin{array}{c}640 \\
(58.2)\end{array}$ & $\begin{array}{c}271 \\
(56.9)\end{array}$ & $\begin{array}{c}172 \\
(51.7)\end{array}$ & $\begin{array}{c}171 \\
(62.0)\end{array}$ & $\begin{array}{c}108 \\
(53.2)\end{array}$ & $\begin{array}{c}145 \\
(48.7)\end{array}$ & $\begin{array}{c}279 \\
(50.9)\end{array}$ & 1,786 & 3,231 & 55.2 \\
\hline Never smokers & $\begin{array}{c}927 \\
(85.4)\end{array}$ & - & $\begin{array}{c}265 \\
(79.6)\end{array}$ & - & - & - & $\begin{array}{c}452 \\
(83.1)\end{array}$ & 1,644 & 1,962 & 83.80 \\
\hline Current smokers & $\begin{array}{c}137 \\
(12.6)\end{array}$ & - & $\begin{array}{l}26 \\
(7.8)\end{array}$ & $\begin{array}{c}12 \\
(4.0)\end{array}$ & - & - & $\begin{array}{c}41 \\
(7.5)\end{array}$ & 216 & 2,236 & 9.66 \\
\hline \multicolumn{11}{|l|}{$\begin{array}{l}\text { List of clinical } \\
\text { symptoms COVID-19 }\end{array}$} \\
\hline Fever & $\begin{array}{c}966 \\
(87.9)\end{array}$ & $\begin{array}{c}390 \\
(85.9)\end{array}$ & $\begin{array}{c}207 \\
(62.2)\end{array}$ & $\begin{array}{c}249 \\
(91.0)\end{array}$ & $\begin{array}{c}181 \\
(89.2)\end{array}$ & $\begin{array}{c}218 \\
(73.0)\end{array}$ & $\begin{array}{c}476 \\
(95.2)\end{array}$ & 2,687 & 3,231 & 85.00 \\
\hline Cough & $\begin{array}{c}744 \\
(67.7)\end{array}$ & $\begin{array}{c}269 \\
(59.4)\end{array}$ & $\begin{array}{c}106 \\
(31.8)\end{array}$ & $\begin{array}{c}185 \\
(68.0)\end{array}$ & $\begin{array}{c}122 \\
(60.1)\end{array}$ & $\begin{array}{c}105 \\
(35.0)\end{array}$ & $\begin{array}{c}415 \\
(75.7)\end{array}$ & 1,946 & 2,755 & 70.63 \\
\hline Chest tightness & - & - & - & $\begin{array}{c}103 \\
(38.0)\end{array}$ & $\begin{array}{c}72 \\
(35.5)\end{array}$ & - & $\begin{array}{c}162 \\
(38.1)\end{array}$ & 337 & 902 & 37.36 \\
\hline Expectoration & $\begin{array}{c}367 \\
(33.4)\end{array}$ & $\begin{array}{c}161 \\
(35.5)\end{array}$ & - & $\begin{array}{c}83 \\
(30.0)\end{array}$ & - & - & - & 611 & 1,836 & 33.27 \\
\hline Fatigue & $\begin{array}{c}419 \\
(38.1)\end{array}$ & - & $40(12.0)$ & $\begin{array}{c}137 \\
(50.0)\end{array}$ & $\begin{array}{c}16 \\
(7.9)\end{array}$ & $\begin{array}{c}13 \\
(4.4)\end{array}$ & $\begin{array}{c}258 \\
(47.1)\end{array}$ & 883 & 2,755 & 32.05 \\
\hline Dyspnea & - & - & $\begin{array}{c}1 \\
(0.3)\end{array}$ & $\begin{array}{c}120 \\
(44.0)\end{array}$ & $\begin{array}{c}3 \\
(1.5)\end{array}$ & - & $\begin{array}{c}310 \\
(56.6)\end{array}$ & 434 & 1,358 & 31.95 \\
\hline Shortness of breath & $204(18.6)$ & $\begin{array}{c}109 \\
(24.4)\end{array}$ & - & $\begin{array}{c}120 \\
(44.0)\end{array}$ & $\begin{array}{c}59 \\
(29.1)\end{array}$ & - & $\begin{array}{c}310 \\
(56.6)\end{array}$ & 802 & 2,571 & 31.19 \\
\hline Myalgia or arthralgia & $\begin{array}{c}163 \\
(14.8)\end{array}$ & $\begin{array}{c}55 \\
(12.6)\end{array}$ & - & $\begin{array}{c}60 \\
(22.0)\end{array}$ & $\begin{array}{c}54 \\
(26.6)\end{array}$ & - & $\begin{array}{c}111 \\
(20.3)\end{array}$ & 443 & 2,860 & 15.48 \\
\hline Diarrhea & $\begin{array}{c}41 \\
(3.7)\end{array}$ & - & $\begin{array}{c}7 \\
(2.1)\end{array}$ & $\begin{array}{c}77 \\
(28.0)\end{array}$ & $\begin{array}{c}10 \\
(4.9)\end{array}$ & $\begin{array}{c}9 \\
(3.0)\end{array}$ & $\begin{array}{c}179 \\
(32.7)\end{array}$ & 314 & 2,755 & 11.39 \\
\hline Headache & $\begin{array}{c}150 \\
(13.6)\end{array}$ & - & $\begin{array}{c}30 \\
(9.0)\end{array}$ & $\begin{array}{c}31 \\
(11.0)\end{array}$ & $\begin{array}{c}10 \\
(4.9)\end{array}$ & $\begin{array}{c}5 \\
(1.7)\end{array}$ & $\begin{array}{c}62 \\
(11.3)\end{array}$ & 288 & 2,755 & 10.45 \\
\hline Chill & $\begin{array}{c}125 \\
(11.4)\end{array}$ & - & $\begin{array}{c}20 \\
(6.0)\end{array}$ & - & - & - & - & 145 & 1,432 & 10.12 \\
\hline Sore throat & $\begin{array}{c}153 \\
(13.9)\end{array}$ & - & - & - & - & $\begin{array}{c}2 \\
(0.7)\end{array}$ & $\begin{array}{c}28 \\
(5.1)\end{array}$ & 183 & 1,945 & 9.40 \\
\hline Confusion & - & $\begin{array}{c}35 \\
(8.1)\end{array}$ & $\begin{array}{c}31 \\
(9.3)\end{array}$ & $\begin{array}{c}12 \\
(4.0)\end{array}$ & - & - & - & 78 & 1,040 & 7.50 \\
\hline Nasal congestion & $\begin{array}{c}53 \\
(4.8)\end{array}$ & - & $\begin{array}{c}13 \\
(3.9)\end{array}$ & - & - & $\begin{array}{c}3 \\
(1.0)\end{array}$ & - & 69 & 1,730 & 3.98 \\
\hline Pharyngodynia & - & $\begin{array}{c}35 \\
(8.1)\end{array}$ & $\begin{array}{c}31 \\
(9.3)\end{array}$ & $\begin{array}{c}12 \\
(4.0)\end{array}$ & - & - & - & 78 & 1,040 & 7.50 \\
\hline
\end{tabular}




\section{Obstetrics \& Gynecology Science}

Harsh Goel, et al. Review of 3,000 COVID-19 cases

Table 1. Continued

\begin{tabular}{|c|c|c|c|c|c|c|c|c|c|c|}
\hline Parameter & $\begin{array}{c}\text { Guan et } \\
\text { al. }[4] \\
(n=1,099)\end{array}$ & $\begin{array}{l}\text { Feng et } \\
\text { al. [18] } \\
(n=476)\end{array}$ & $\begin{array}{c}\text { Yu et } \\
\text { al. [19] } \\
(n=333)\end{array}$ & $\begin{array}{l}\text { Chen et } \\
\text { al. [20] } \\
(n=274)\end{array}$ & $\begin{array}{l}\text { Chen et } \\
\text { al. [21] } \\
(n=203)\end{array}$ & $\begin{array}{l}\text { Cai et } \\
\text { al. [22] } \\
(n=298)\end{array}$ & $\begin{array}{c}\text { Li et } \\
\text { al. [23] } \\
(n=548)\end{array}$ & $\begin{array}{l}\text { Events } \\
\text { number }\end{array}$ & Total & $\begin{array}{c}\text { Overall } \\
\text { percentage }\end{array}$ \\
\hline Dizziness & - & - & $\begin{array}{c}8 \\
(2.4)\end{array}$ & $\begin{array}{c}21 \\
(8.0)\end{array}$ & $\begin{array}{c}4 \\
(2.0)\end{array}$ & - & $\begin{array}{c}56 \\
(10.2)\end{array}$ & 89 & 1,358 & 6.55 \\
\hline Shivering & - & $\begin{array}{c}24 \\
(6.4)\end{array}$ & $\begin{array}{c}19 \\
(5.7)\end{array}$ & - & - & - & - & 43 & 707 & 6.08 \\
\hline Anorexia & - & $\begin{array}{c}24 \\
(6.4)\end{array}$ & $\begin{array}{c}19 \\
(5.7)\end{array}$ & - & - & - & - & 43 & 707 & 6.08 \\
\hline Nausea or vomiting & $55(5.0)$ & - & - & $\begin{array}{c}24 \\
(9.0)\end{array}$ & $\begin{array}{c}3 \\
(1.5)\end{array}$ & - & $\begin{array}{c}45 \\
(8.2)\end{array}$ & 127 & 2,124 & 5.97 \\
\hline Chest pain & - & $\begin{array}{c}21 \\
(4.4)\end{array}$ & - & - & $\begin{array}{c}4 \\
(2.0)\end{array}$ & - & $\begin{array}{c}41 \\
(7.5)\end{array}$ & 66 & 1,191 & 5.54 \\
\hline Abdominal pain & - & - & - & $\begin{array}{c}19 \\
(7.0)\end{array}$ & $\begin{array}{c}4 \\
(2.0)\end{array}$ & - & $\begin{array}{c}16 \\
(2.9)\end{array}$ & 39 & 1,025 & 3.80 \\
\hline Hemoptysis & $\begin{array}{c}10 \\
(0.9)\end{array}$ & $\begin{array}{c}5 \\
(1.1)\end{array}$ & - & $\begin{array}{c}7 \\
(3.0)\end{array}$ & - & - & - & 22 & 1,808 & 1.21 \\
\hline
\end{tabular}

Data are presented as number of patients (\%) or median (range) unless otherwise indicated.

COVID-19, coronavirus disease 2019.

Table 2. Co-morbidities of patients with COVID-19

\begin{tabular}{|c|c|c|c|c|c|c|c|c|c|c|}
\hline $\begin{array}{l}\text { Co-morbid } \\
\text { conditions }\end{array}$ & $\begin{array}{c}\text { Guan et } \\
\text { al. }[4] \\
(n=1,099)\end{array}$ & $\begin{array}{l}\text { Feng et } \\
\text { al. [18] } \\
(n=476)\end{array}$ & $\begin{array}{c}\text { Yu et al. } \\
{[19]} \\
(n=333)\end{array}$ & $\begin{array}{l}\text { Chen et } \\
\text { al. [20] } \\
(n=274)\end{array}$ & $\begin{array}{l}\text { Chen et } \\
\text { al. [21] } \\
(n=203)\end{array}$ & $\begin{array}{c}\text { Cai et } \\
\text { al. [22] } \\
(n=298)\end{array}$ & $\begin{array}{c}\text { Li et al. } \\
{[23]} \\
(n=548)\end{array}$ & $\begin{array}{l}\text { Events } \\
\text { number }\end{array}$ & $\begin{array}{c}\text { Total } \\
\text { number }\end{array}$ & $\begin{array}{l}\text { Overall } \\
\text { percent }\end{array}$ \\
\hline Hypertension & $\begin{array}{c}164 \\
(14.9)\end{array}$ & $\begin{array}{c}113 \\
(23.7)\end{array}$ & - & $\begin{array}{c}93 \\
(34.0)\end{array}$ & $\begin{array}{c}43 \\
(21.2)\end{array}$ & $\begin{array}{c}47 \\
(15.8)\end{array}$ & $\begin{array}{c}166 \\
(30.3)\end{array}$ & 626 & 2,898 & 21.60 \\
\hline Diabetes & $\begin{array}{c}81 \\
(7.4)\end{array}$ & $\begin{array}{c}49 \\
(10.0)\end{array}$ & $\begin{array}{c}28 \\
(8.4)\end{array}$ & $\begin{array}{c}47 \\
(17.0)\end{array}$ & $\begin{array}{c}16 \\
(7.9)\end{array}$ & $\begin{array}{c}18 \\
(6.0)\end{array}$ & $\begin{array}{c}83 \\
(15.0)\end{array}$ & 322 & 3,231 & 9.96 \\
\hline Cardiovascular disease & $\begin{array}{l}27 \\
(2.5)\end{array}$ & $38(8.0)$ & $\begin{array}{l}24 \\
(7.2)\end{array}$ & $\begin{array}{c}23 \\
(8.0)\end{array}$ & $\begin{array}{c}16 \\
(7.9)\end{array}$ & $\begin{array}{c}25 \\
(8.4)\end{array}$ & $\begin{array}{c}34 \\
(6.2)\end{array}$ & 187 & 3,231 & 5.78 \\
\hline $\begin{array}{l}\text { Chronic obstructive } \\
\text { pulmonary disease }\end{array}$ & $\begin{array}{c}12 \\
(1.1)\end{array}$ & $\begin{array}{c}22 \\
(4.6)\end{array}$ & - & $\begin{array}{c}18 \\
(7.0)\end{array}$ & $\begin{array}{c}8 \\
(3.9)\end{array}$ & - & $\begin{array}{c}17 \\
(3.1)\end{array}$ & 77 & 2,600 & 2.96 \\
\hline Cancer & $\begin{array}{c}10 \\
(0.9)\end{array}$ & $\begin{array}{c}12 \\
(2.5)\end{array}$ & - & $\begin{array}{c}7 \\
(3.0)\end{array}$ & $\begin{array}{c}7 \\
(3.4)\end{array}$ & $\begin{array}{c}4 \\
(1.3)\end{array}$ & $\begin{array}{c}24 \\
(4.7)\end{array}$ & 64 & 2,863 & 2.23 \\
\hline Cerebrovascular diseases & $\begin{array}{c}15 \\
(1.4)\end{array}$ & $\begin{array}{c}17 \\
(3.6)\end{array}$ & - & $\begin{array}{c}4 \\
(1.0)\end{array}$ & $\begin{array}{c}9 \\
(4.4)\end{array}$ & - & - & 45 & 2,052 & 2.19 \\
\hline Chronic renal diseases & $\begin{array}{c}8 \\
(0.7)\end{array}$ & $\begin{array}{c}4 \\
(0.8)\end{array}$ & - & $\begin{array}{c}4 \\
(1.0)\end{array}$ & $\begin{array}{c}8 \\
(3.9)\end{array}$ & - & $\begin{array}{c}10 \\
(1.8)\end{array}$ & 34 & 2,599 & 1.30 \\
\hline
\end{tabular}

Data are presented as number of patients (\%) unless otherwise indicated.

COVID-19, coronavirus disease 2019.

conditions analyzed were hypertension (21.60\%), diabetes $(9.96 \%)$, cardiovascular disease $(5.78 \%)$, chronic pulmonary obstructive disease $(2.96 \%)$, malignancy $(2.23 \%)$, cerebrovascular disease $(2.19 \%)$, and chronic nephropathy $(1.30 \%)$ were investigated in this study. Although the number of subjects was higher in the study by Guan et al. [4], the associated comorbid conditions were not so prevalent; this could be because the subjects were relatively younger, with the lowest median age of 47 years, compared with those in the other studies [18-23]. 


\section{Obstetrics \& Gynecology Science}

Vol. 64, No. 2, 2021

Table 3. Hematological biochemical and inflammatory parameters profile

\begin{tabular}{|c|c|c|c|c|c|c|c|c|c|c|}
\hline & $\begin{array}{c}\text { Guan et al. } \\
{[4]} \\
(n=1,099)\end{array}$ & $\begin{array}{l}\text { Feng et } \\
\text { al. [18] } \\
(n=476)\end{array}$ & $\begin{array}{c}\text { Yu et al. } \\
{[19]} \\
(n=333)\end{array}$ & $\begin{array}{c}\text { Chen et al. } \\
{[20]} \\
(n=274)\end{array}$ & $\begin{array}{l}\text { Chen et } \\
\text { al. [21] } \\
(n=203)\end{array}$ & $\begin{array}{l}\text { Cai et } \\
\text { al. [22] } \\
(n=298)\end{array}$ & $\begin{array}{l}\text { Li et al. } \\
{[23]} \\
(n=548)\end{array}$ & $\begin{array}{l}\text { Events } \\
\text { number }\end{array}$ & $\begin{array}{c}\text { Total } \\
\text { number }\end{array}$ & $\begin{array}{l}\text { Overall } \\
\text { percent }\end{array}$ \\
\hline \multicolumn{11}{|l|}{$\begin{array}{c}\text { Hematological } \\
\text { parameters }\end{array}$} \\
\hline $\begin{array}{l}\text { Haemoglobin } \\
(\mathrm{g} / \mathrm{L})\end{array}$ & $\begin{array}{c}134.0 \\
(119.0-148.0)\end{array}$ & $\begin{array}{c}132 \\
(121-144)\end{array}$ & - & $\begin{array}{c}128.0 \\
(116.0-140.0)\end{array}$ & - & - & - & - & - & - \\
\hline $\begin{array}{l}\text { Platelets } \\
\text { (decreased) }\end{array}$ & $\begin{array}{c}315 / 869 \\
(36.2)\end{array}$ & - & - & - & $\begin{array}{c}22 / 203 \\
(10.8)\end{array}$ & - & $\begin{array}{c}157 / 539 \\
(29.1)\end{array}$ & 494 & 1,611 & 28.80 \\
\hline $\begin{array}{l}\text { TLC } \\
\text { (decreased) }\end{array}$ & $\begin{array}{c}330 / 978 \\
(33.7)\end{array}$ & $\begin{array}{c}91 / 475 \\
(19.2)\end{array}$ & $\begin{array}{c}56 / 333 \\
(18.6)\end{array}$ & $\begin{array}{c}58 / 274 \\
(21.0)\end{array}$ & $\begin{array}{c}79 / 203 \\
(38.9)\end{array}$ & $\begin{array}{c}114 / 298 \\
(38.9)\end{array}$ & $\begin{array}{c}130 / 542 \\
(24.0)\end{array}$ & 858 & 3,098 & 27.69 \\
\hline $\begin{array}{l}\text { TLC } \\
\text { (increased) }\end{array}$ & $\begin{array}{c}58 / 978 \\
(5.9)\end{array}$ & $\begin{array}{c}49 / 475 \\
(10.3)\end{array}$ & $\begin{array}{l}1 / 333 \\
(0.3)\end{array}$ & $\begin{array}{c}62 / 274 \\
(23.0)\end{array}$ & $\begin{array}{c}14 / 203 \\
(6.9)\end{array}$ & & $\begin{array}{c}63 / 542 \\
(11.6)\end{array}$ & 247 & 2,805 & 8.80 \\
\hline $\begin{array}{l}\text { Lymphocytes } \\
\text { (decreased) }\end{array}$ & $\begin{array}{c}731 / 890 \\
(82.1)\end{array}$ & $\begin{array}{c}225 / 476 \\
(47.3)\end{array}$ & $\begin{array}{c}28 / 333 \\
(10.8)\end{array}$ & $\begin{array}{c}179 / 274 \\
(65.32)\end{array}$ & $\begin{array}{c}117 / 203 \\
(57.6)\end{array}$ & - & $\begin{array}{c}489 / 542 \\
(90.2)\end{array}$ & 1,769 & 2,718 & 65.08 \\
\hline $\begin{array}{l}\text { Neutrophils } \\
\text { (increased) }\end{array}$ & - & - & $\begin{array}{l}1 / 333 \\
(0.4)\end{array}$ & $\begin{array}{c}93 / 274 \\
(34.0)\end{array}$ & - & - & $\begin{array}{c}118 / 542 \\
(21.8)\end{array}$ & 212 & 1,149 & 18.45 \\
\hline \multicolumn{11}{|l|}{$\begin{array}{c}\text { Biochemical } \\
\text { parameters } \\
\text { (increased) }\end{array}$} \\
\hline $\begin{array}{l}\text { Albumin } \\
\text { (increased) }\end{array}$ & - & - & - & $\begin{array}{c}96 / 274 \\
(35.0)\end{array}$ & $\begin{array}{c}131 / 203 \\
(64.5)\end{array}$ & - & $\begin{array}{c}320 / 541 \\
(59.1)\end{array}$ & 547 & 1,018 & 53.72 \\
\hline $\begin{array}{l}\text { LDH } \\
\text { (increased) }\end{array}$ & $\begin{array}{c}277 / 675 \\
(41.0)\end{array}$ & - & - & $\begin{array}{c}116 / 274 \\
(42.0)\end{array}$ & $\begin{array}{c}87 / 203 \\
(42.9)\end{array}$ & $\begin{array}{c}23 / 274 \\
(8.4)\end{array}$ & $\begin{array}{c}393 / 534 \\
(73.6)\end{array}$ & 896 & 1,960 & 45.71 \\
\hline $\begin{array}{l}\text { Natriureti } \\
\text { peptide } \\
\text { (increased) }\end{array}$ & - & - & - & 85/173 (49.0) & - & - & $\begin{array}{l}92 / 33 \\
(27.5)\end{array}$ & 177 & 508 & 34.84 \\
\hline $\begin{array}{l}\text { Globulin } \\
\text { (increased) }\end{array}$ & - & - & - & - & $\begin{array}{c}68 / 203 \\
(33.5)\end{array}$ & - & $\begin{array}{c}218 / 540 \\
(40.4)\end{array}$ & 68 & 203 & 33.5 \\
\hline $\begin{array}{l}\text { AST } \\
\text { (increased) }\end{array}$ & $\begin{array}{c}168 / 757 \\
(22.2)\end{array}$ & - & - & $\begin{array}{c}84 / 274 \\
(31.0)\end{array}$ & $\begin{array}{c}69 / 203 \\
(34.0)\end{array}$ & $\begin{array}{c}25 / 298 \\
(8.4)\end{array}$ & $\begin{array}{c}179 / 540 \\
(33.1)\end{array}$ & 525 & 2,072 & 25.33 \\
\hline $\begin{array}{l}\text { ALT } \\
\quad \text { (increased) }\end{array}$ & $\begin{array}{c}158 / 741 \\
(21.3)\end{array}$ & - & - & $60 / 274(22.0)$ & $\begin{array}{c}30 / 203 \\
(14.8)\end{array}$ & $\begin{array}{c}39 / 298 \\
(13.1)\end{array}$ & $\begin{array}{c}125 / 541 \\
(23.1)\end{array}$ & 412 & 1,884 & 21.86 \\
\hline $\begin{array}{l}\text { BUN } \\
\text { (increased) }\end{array}$ & - & - & - & & - & - & $\begin{array}{c}85 / 539 \\
(15.8)\end{array}$ & 85 & 539 & 15.8 \\
\hline $\begin{array}{l}\text { Creatinine } \\
\text { (increased) }\end{array}$ & $\begin{array}{c}12 / 752 \\
(1.6)\end{array}$ & - & - & - & $\begin{array}{c}25 / 203 \\
(12.3)\end{array}$ & - & $\begin{array}{c}146 / 539 \\
(27.1)\end{array}$ & 183 & 1,494 & 12.24 \\
\hline $\begin{array}{l}\text { Total bilirubing } \\
\text { (increased) }\end{array}$ & $76 / 722(10.5)$ & - & - & - & - & $\begin{array}{c}24 / 298 \\
(8.1)\end{array}$ & $\begin{array}{c}24 / 541 \\
(4.4)\end{array}$ & 124 & 1,561 & 7.94 \\
\hline \multicolumn{11}{|l|}{$\begin{array}{l}\text { Inflammatory } \\
\text { cytokines } \\
\text { (increased) }\end{array}$} \\
\hline $\begin{array}{l}\text { C-reactive } \\
\text { protein level }\end{array}$ & $\begin{array}{c}481 / 793 \\
(60.7)\end{array}$ & $\begin{array}{c}266 / 41 \\
(64.1)\end{array}$ & $\begin{array}{c}84 / 333 \\
(42.9)\end{array}$ & $80 / 243(33.0)$ & $\begin{array}{c}110 / 203 \\
(54.2)\end{array}$ & $\begin{array}{c}196 / 291 \\
(67.4)\end{array}$ & $\begin{array}{c}460 / 540 \\
(85.2)\end{array}$ & 1,677 & 2,818 & 59.51 \\
\hline $\begin{array}{l}\text { Tumor necrosis } \\
\text { factor }\end{array}$ & - & - & - & $93 / 163(57.0)$ & - & - & $\begin{array}{c}182 / 309 \\
(58.9)\end{array}$ & 275 & 472 & 58.26 \\
\hline $\begin{array}{l}\text { Erythrocyte } \\
\text { sedimentation } \\
\text { rate }\end{array}$ & - & - & - & - & $\begin{array}{c}82 / 203 \\
(40.4)\end{array}$ & $\begin{array}{c}117 / 287 \\
(40.8)\end{array}$ & $\begin{array}{c}377 / 518 \\
(72.8)\end{array}$ & 576 & 1,008 & 57.14 \\
\hline
\end{tabular}




\section{Obstetrics \& Gynecology Science}

Harsh Goel, et al. Review of 3,000 COVID-19 cases

Table 3. Continued

\begin{tabular}{|c|c|c|c|c|c|c|c|c|c|c|}
\hline & $\begin{array}{c}\text { Guan et al. } \\
{[4]} \\
(n=1,099)\end{array}$ & $\begin{array}{l}\text { Feng et } \\
\text { al. [18] } \\
(n=476)\end{array}$ & $\begin{array}{c}\text { Yu et al. } \\
{[19]} \\
(n=333)\end{array}$ & $\begin{array}{c}\text { Chen et al. } \\
\text { [20] } \\
(n=274)\end{array}$ & $\begin{array}{l}\text { Chen et } \\
\text { al. [21] } \\
(n=203)\end{array}$ & $\begin{array}{l}\text { Cai et } \\
\text { al. [22] } \\
(n=298)\end{array}$ & $\begin{array}{c}\text { Li et al. } \\
{[23]} \\
(n=548)\end{array}$ & $\begin{array}{l}\text { Events } \\
\text { number }\end{array}$ & $\begin{array}{c}\text { Total } \\
\text { number }\end{array}$ & $\begin{array}{l}\text { Overall } \\
\text { percent }\end{array}$ \\
\hline Interleukin-6 & - & - & - & $\begin{array}{c}119 / 163 \\
(73.0)\end{array}$ & $\begin{array}{c}44 / 203 \\
(21.7)\end{array}$ & $\begin{array}{c}146 / 294 \\
(49.7)\end{array}$ & $\begin{array}{c}221 / 312 \\
(70.8)\end{array}$ & 530 & 972 & 54.52 \\
\hline Interleukin-2 & - & - & - & $\begin{array}{c}84 / 163 \\
(52.0)\end{array}$ & - & - & $\begin{array}{c}164 / 309 \\
(53.1)\end{array}$ & 248 & 472 & 52.54 \\
\hline Interleukin-10 & - & - & - & $\begin{array}{c}58 / 163 \\
(36.0)\end{array}$ & - & - & $\begin{array}{c}83 / 307 \\
(27.0)\end{array}$ & 141 & 470 & 30.00 \\
\hline Interleukin-8 & - & - & - & $\begin{array}{c}24 / 163 \\
(15.0)\end{array}$ & - & - & $\begin{array}{c}24 / 309 \\
(7.8)\end{array}$ & 48 & 472 & 10.16 \\
\hline $\begin{array}{l}\text { Procalcitonin } \\
\text { level }\end{array}$ & - & - & - & $\begin{array}{c}8 / 236 \\
(3.0)\end{array}$ & $\begin{array}{c}7 / 203 \\
(3.4)\end{array}$ & - & $\begin{array}{c}46 / 486 \\
(9.5)\end{array}$ & 61 & 925 & 6.59 \\
\hline
\end{tabular}

Data are presented as number of patients (\%) or mean (range) unless otherwise indicated.

TLC, total leukocytes count; LDH, lactate dehyadrogenase, AST, aspartate transaminase; ALT, alanine transaminase; BUN, blood urea nitrogen.

Table 4. Complications, treatment and outcomes of patients with COVID-19

\begin{tabular}{|c|c|c|c|c|c|c|c|c|c|}
\hline Characteristic & $\begin{array}{c}\text { Guan et al. } \\
{[4]} \\
(n=1,099)\end{array}$ & $\begin{array}{l}\text { Feng et } \\
\text { al. [18] } \\
(n=476)\end{array}$ & $\begin{array}{l}\text { Chen et } \\
\text { al. [20] } \\
(n=274)\end{array}$ & $\begin{array}{l}\text { Chen et } \\
\text { al. [21] } \\
(n=203)\end{array}$ & $\begin{array}{c}\text { Cai et al. } \\
{[22]} \\
(n=298)\end{array}$ & $\begin{array}{c}\text { Li et } \\
\text { al. [23] } \\
(n=548)\end{array}$ & $\begin{array}{c}\text { Total } \\
\text { events } \\
\text { number }\end{array}$ & $\begin{array}{c}\text { Total } \\
\text { number }\end{array}$ & $\begin{array}{l}\text { Overall } \\
\text { percent }\end{array}$ \\
\hline Oxygen therapy & $418(38.0)$ & $36(77.0)$ & $251(92.0)$ & $123(60.6)$ & - & $355(64.8)$ & 1,515 & 2,600 & 58.26 \\
\hline Antiviral therapy & $393(35.8)$ & $286(60.1)$ & $236(86.0)$ & $131(64.8)$ & $236(79.2)$ & $398(72.6)$ & 1,680 & 2,898 & 57.97 \\
\hline Intravenous antibiotics & $632(57.5)$ & $319(67.0)$ & $249(91.0)$ & - & - & $7(1.3)$ & 1,207 & 2,397 & 50.35 \\
\hline Systemic corticosteroids & $204(18.6)$ & $127(26.0)$ & - & $107(52.7)$ & $91(30.5)$ & $341(62.2)$ & 870 & 2,624 & 33.15 \\
\hline Intravenous immunoglobin & $143(13.0)$ & - & $54(20.0)$ & - & $94(31.5)$ & $213(38.9)$ & 504 & 2,219 & 22.71 \\
\hline Mechanical ventilation & $67(6.1)$ & - & $119(43.0)$ & $39(19.2)$ & $30(10.1)$ & - & 255 & 1,874 & 13.60 \\
\hline Invasive & $24(2.2)$ & $39(8.2)$ & $102(37.0)$ & - & - & $25(4.6)$ & 190 & 2,397 & 7.92 \\
\hline Non-invasive & $56(5.1)$ & $34(7.1)$ & $17(6.0)$ & - & - & $78(14.2)$ & 185 & 2,397 & 7.71 \\
\hline Concurrent chemoradiation & $9(0.8)$ & - & $3(1.0)$ & - & - & $2(0.4)$ & 14 & 1,921 & 0.72 \\
\hline $\begin{array}{l}\text { Extracorporealmembrane } \\
\text { oxygenation }\end{array}$ & $5(0.5)$ & $4(0.8)$ & $1(<1)$ & - & $3(1.0)$ & - & 13 & 1,873 & 0.69 \\
\hline Cardiac complications & - & - & $89(44.0)$ & - & - & $119(21.7)$ & 208 & 751 & 27.69 \\
\hline ARDS & $37(3.4)$ & - & $196(72.0)$ & - & - & $210(38.3)$ & 443 & 1,921 & 23.06 \\
\hline Liver dysfunction & - & - & $13(5.0)$ & - & - & $106(19.3)$ & 119 & 822 & 14.47 \\
\hline Acute kidney injury & $6(0.5)$ & - & $29(11.0)$ & - & - & $95 /(17.3)$ & 130 & 1,921 & 6.76 \\
\hline Septic shock & $11(1.0)$ & - & $46(17.0)$ & - & - & - & 57 & 1,373 & 4.15 \\
\hline DIC & $1(0.1)$ & - & $21(8.0)$ & - & - & - & 22 & 1,373 & 1.60 \\
\hline Staying in hospital & $1,029(93.6)$ & $23(4.8)$ & - & - & - & $168(30.8)$ & 1,220 & 2,120 & 57.54 \\
\hline Discharge from hospital & $55(5.0)$ & $403(84.7)$ & - & - & $268(89.9)$ & $287(52.7)$ & 1,013 & 2,418 & 41.89 \\
\hline Death & $15(1.4)$ & $38(8.0)$ & - & $26(12.8)$ & $3(1.0)$ & $90(16.5)$ & 172 & 2,621 & 6.56 \\
\hline
\end{tabular}

Data are presented as number of patients (\%) unless otherwise indicated.

COVID-19, coronavirus disease 2019; ARDS, acute respiratory distress syndrome; DIC, disseminated intravascular coagulopathy. 


\section{Obstetrics \& Gynecology Science}

Vol. 64, No. 2, 2021

Table 5. Maternal \& neonatal outcome of COVID-19 in pregnant women

\begin{tabular}{|c|c|c|c|c|c|c|c|c|}
\hline Clinical characteristic & $\begin{array}{c}\text { Juan et al. } \\
\text { [24] } \\
(n=324)\end{array}$ & $\begin{array}{c}\text { Galang et } \\
\text { al. [25] } \\
(n=98)\end{array}$ & $\begin{array}{c}\text { Yoon et al. } \\
{[26]} \\
(n=201)\end{array}$ & $\begin{array}{l}\text { Trippella } \\
\text { et al. [27] } \\
(n=275)\end{array}$ & $\begin{array}{l}\text { Smith et } \\
\text { al. [28] } \\
(n=92)\end{array}$ & $\begin{array}{l}\text { Events } \\
\text { number }\end{array}$ & $\begin{array}{c}\text { Total } \\
\text { number }\end{array}$ & $\begin{array}{l}\text { Overall } \\
\text { percent }\end{array}$ \\
\hline \multicolumn{9}{|l|}{$\begin{array}{l}\text { Symptoms of COVID-19 } \\
\text { in pregnant women }\end{array}$} \\
\hline Fever & $\begin{array}{c}138 / 295 \\
(46.8)\end{array}$ & $\begin{array}{l}76 / 92 \\
(83.0)\end{array}$ & $\begin{array}{c}85 / 201 \\
(42.3)\end{array}$ & $\begin{array}{c}155 / 275 \\
(58.0)\end{array}$ & $\begin{array}{c}57 / 92 \\
(61.96)\end{array}$ & 511 & 955 & 53.50 \\
\hline Cough & $\begin{array}{c}101 / 295 \\
(34.2)\end{array}$ & $\begin{array}{l}34 / 66 \\
(52.0)\end{array}$ & $\begin{array}{c}64 / 201 \\
(31.8)\end{array}$ & $\begin{array}{c}95 / 275 \\
(36.0)\end{array}$ & $\begin{array}{c}35 / 92 \\
(38.04)\end{array}$ & 329 & 929 & 35.41 \\
\hline Malaise & 0 & $\begin{array}{l}14 / 31 \\
(45.0)\end{array}$ & $\begin{array}{l}18 / 95 \\
(18.9)\end{array}$ & $\begin{array}{c}37 / 275 \\
(14.0)\end{array}$ & $\begin{array}{c}25 / 82 \\
(30.49)\end{array}$ & 94 & 483 & 19.46 \\
\hline Shortness of breath & $\begin{array}{c}39 / 295 \\
(13.2)\end{array}$ & $\begin{array}{l}12 / 47 \\
(26.0)\end{array}$ & $\begin{array}{c}16 / 142 \\
(11.3)\end{array}$ & - & $\begin{array}{c}10 / 83 \\
(12.05)\end{array}$ & 77 & 567 & 13.58 \\
\hline Myalgia & $\begin{array}{l}27 / 295 \\
(9.2)\end{array}$ & $\begin{array}{l}5 / 21 \\
(24.0)\end{array}$ & $\begin{array}{l}15 / 56 \\
(21.4)\end{array}$ & $\begin{array}{c}37 / 275 \\
(14.0)\end{array}$ & $\begin{array}{l}6 / 28 \\
(21.4)\end{array}$ & 90 & 675 & 13.33 \\
\hline Diarrhea/GI symptoms & $\begin{array}{c}11 / 295 \\
(3.7)\end{array}$ & $\begin{array}{l}76 / 92 \\
(83.0)\end{array}$ & $\begin{array}{c}10 / 134 \\
(7.5)\end{array}$ & $\begin{array}{c}9 / 275 \\
(3.0)\end{array}$ & $\begin{array}{c}4 / 38 \\
(10.43)\end{array}$ & 110 & 834 & 13.18 \\
\hline Fatigue & $\begin{array}{l}28 / 295 \\
(9.5)\end{array}$ & $\begin{array}{l}7 / 29 \\
(24.0)\end{array}$ & - & $\begin{array}{c}28 / 275 \\
(10.0)\end{array}$ & $\begin{array}{c}25 / 82 \\
(30.49)\end{array}$ & 88 & 681 & 12.92 \\
\hline Sore throat & $\begin{array}{c}10 / 295 \\
(3.4)\end{array}$ & $\begin{array}{l}1 / 24 \\
(4.0)\end{array}$ & $\begin{array}{l}8 / 84 \\
(9.5)\end{array}$ & $\begin{array}{c}9 / 275 \\
(3.0)\end{array}$ & $\begin{array}{l}6 / 50 \\
(12.0)\end{array}$ & 34 & 728 & 4.67 \\
\hline \multicolumn{9}{|c|}{$\begin{array}{l}\text { Hemogram findings of } \\
\text { pregnant women with COVID-19 }\end{array}$} \\
\hline TLC (decreased) & $\begin{array}{c}146 / 182 \\
(80.2)\end{array}$ & - & - & - & - & 146 & 182 & 80.2 \\
\hline Lymphocytes (decreased) & $\begin{array}{c}85 / 197 \\
(43.1)\end{array}$ & $\begin{array}{l}27 / 50 \\
(54.0)\end{array}$ & $\begin{array}{c}52 / 120 \\
(43.3)\end{array}$ & $\begin{array}{c}31 / 108 \\
(29.0)\end{array}$ & $\begin{array}{c}46 / 69 \\
(66.67)\end{array}$ & 241 & 544 & 44.30 \\
\hline Thrombocytopenia & - & $\begin{array}{l}8 / 18 \\
(44.0)\end{array}$ & $\begin{array}{l}7 / 31 \\
(22.6)\end{array}$ & - & - & 15 & 49 & 30.61 \\
\hline TLC (increased) & $\begin{array}{c}4 / 19 \\
(21.05)\end{array}$ & $\begin{array}{l}12 / 47 \\
(26.0)\end{array}$ & $\begin{array}{l}28 / 89 \\
(31.5)\end{array}$ & - & - & 40 & 155 & 28.38 \\
\hline \multicolumn{9}{|c|}{$\begin{array}{l}\text { Biochemical of pregnant women } \\
\text { with COVID-19 }\end{array}$} \\
\hline Elevated CRP & $\begin{array}{c}90 / 197 \\
(45.7)\end{array}$ & - & $\begin{array}{c}65 / 103 \\
(63.1)\end{array}$ & $\begin{array}{l}52 / 108 \\
(48.0)\end{array}$ & - & 207 & 408 & 50.73 \\
\hline Elevated AST & $\begin{array}{c}5 / 42 \\
(11.9)\end{array}$ & $\begin{array}{l}7 / 28 \\
(25.0)\end{array}$ & - & $\begin{array}{c}9 / 108 \\
(8.0)\end{array}$ & - & 21 & 178 & 11.79 \\
\hline Elevated ALT & $\begin{array}{l}5 / 42 \\
(11.9)\end{array}$ & $\begin{array}{l}6 / 28 \\
(21.0)\end{array}$ & - & $\begin{array}{c}9 / 108 \\
(8.0)\end{array}$ & - & 20 & 178 & 11.23 \\
\hline \multicolumn{9}{|c|}{$\begin{array}{l}\text { Treatments of pregnant women } \\
\text { with COVID-19 }\end{array}$} \\
\hline Antibiotics & $\begin{array}{c}111 / 157 \\
(70.7)\end{array}$ & $\begin{array}{l}46 / 49 \\
(94.0)\end{array}$ & - & - & - & 157 & 206 & 76.21 \\
\hline Antivirals & $\begin{array}{c}82 / 217 \\
(37.8)\end{array}$ & $\begin{array}{l}43 / 57 \\
(75.0)\end{array}$ & - & - & - & 125 & 274 & 45.62 \\
\hline $\begin{array}{l}\text { Non-invasive mechanical } \\
\text { ventilation }\end{array}$ & $\begin{array}{l}21 / 170 \\
(12.4)\end{array}$ & - & $\begin{array}{c}5 / 50 \\
(10.0)\end{array}$ & - & - & 26 & 220 & 11.81 \\
\hline
\end{tabular}




\section{Obstetrics \& Gynecology Science}

Harsh Goel, et al. Review of 3,000 COVID-19 cases

Table 5. Continued

\begin{tabular}{|c|c|c|c|c|c|c|c|c|}
\hline Clinical characteristic & $\begin{array}{c}\text { Juan et al. } \\
\text { [24] } \\
(n=324)\end{array}$ & $\begin{array}{l}\text { Galang et } \\
\text { al. [25] } \\
(n=98)\end{array}$ & $\begin{array}{c}\text { Yoon et al. } \\
{[26]} \\
(n=201)\end{array}$ & $\begin{array}{c}\text { Trippella } \\
\text { et al. [27] } \\
(n=275)\end{array}$ & $\begin{array}{l}\text { Smith et } \\
\text { al. [28] } \\
(n=92)\end{array}$ & $\begin{array}{l}\text { Events } \\
\text { number }\end{array}$ & $\begin{array}{c}\text { Total } \\
\text { number }\end{array}$ & $\begin{array}{l}\text { Overall } \\
\text { percent }\end{array}$ \\
\hline ICU admission & $\begin{array}{c}12 / 253 \\
(4.7)\end{array}$ & - & $\begin{array}{c}5 / 103 \\
(4.3)\end{array}$ & $\begin{array}{c}10 / 275 \\
(3.0)\end{array}$ & - & 27 & 631 & 4.27 \\
\hline Invasive mechanical ventilation & $\begin{array}{l}3 / 170 \\
(1.8)\end{array}$ & - & - & $\begin{array}{l}5 / 275 \\
(2.0)\end{array}$ & - & 8 & 445 & 1.79 \\
\hline \multicolumn{9}{|c|}{ Maternal outcomes and complications } \\
\hline Gestational age at delivery (wk) & $28-41$ & 37 & $30-41$ & 37 & 37 & - & - & - \\
\hline Delivery at $<37$ weeks & $\begin{array}{l}72 / 295 \\
(24.4)\end{array}$ & $\begin{array}{l}35 / 94 \\
(61.0)\end{array}$ & $\begin{array}{l}48 / 185 \\
(25.94)\end{array}$ & $\begin{array}{c}48 / 208 \\
(23.0)\end{array}$ & $\begin{array}{l}30 / 47 \\
(63.8)\end{array}$ & 233 & 829 & 28.10 \\
\hline Caesarean delivery & $\begin{array}{l}171 / 219 \\
(78.1)\end{array}$ & $\begin{array}{c}72 / 84 \\
(85.71)\end{array}$ & $\begin{array}{l}163 / 185 \\
(88.10)\end{array}$ & $\begin{array}{l}179 / 239 \\
(75.0)\end{array}$ & $\begin{array}{l}40 / 43 \\
(93.0)\end{array}$ & 553 & 691 & 77.07 \\
\hline Vaginal delivery & $\begin{array}{c}48 / 219 \\
(21.9)\end{array}$ & $\begin{array}{c}12 / 84 \\
(14.28)\end{array}$ & $\begin{array}{l}22 / 185 \\
(11.89)\end{array}$ & $\begin{array}{c}60 / 239 \\
(25.0)\end{array}$ & $\begin{array}{l}03 / 43 \\
(6.97)\end{array}$ & 133 & 691 & 21.93 \\
\hline Maternal mortality & 0 & - & $\begin{array}{l}1 / 100 \\
(1.0)\end{array}$ & $\begin{array}{l}1 / 275 \\
(0.03)\end{array}$ & 0 & 2 & 375 & 0.53 \\
\hline \multicolumn{9}{|l|}{$\begin{array}{l}\text { Neonatal outcomes of pregnancies } \\
\text { with COVID-19 }\end{array}$} \\
\hline 1-minute Apgar score $(>7)$ & All (100) & - & All (100) & $\begin{array}{l}145 / 190 \\
(97.0)\end{array}$ & All (100) & - & - & - \\
\hline 5-minutes Apgar score $(>7)$ & All (100) & - & All (100) & $\begin{array}{c}185 / 190 \\
(76.0)\end{array}$ & All (100) & - & - & - \\
\hline $\begin{array}{l}\text { Mean birth weight at term@ } \\
\text { term delivery }\end{array}$ & - & 3,250 & $1,880-4,050$ & 2,913 & $\begin{array}{l}9 / 21(42.86) \\
\quad<2,500\end{array}$ & - & - & - \\
\hline Transferred-NICU & $\begin{array}{c}49 / 173 \\
(28.3)\end{array}$ & - & - & - & $\begin{array}{c}11 / 13 \\
(76.92)\end{array}$ & 60 & 186 & 32.25 \\
\hline Neonatal asphyxia & $\begin{array}{c}1 / 161 \\
(0.6)\end{array}$ & - & $\begin{array}{c}1 / 177 \\
(0.6)\end{array}$ & - & - & 4 & 329 & 1.21 \\
\hline COVID-19 infection & $3 / 155$ & $1 / 24$ & $4 / 201$ & $16 / 248$ & $1 / 37$ & 25 & 665 & 3.75 \\
\hline
\end{tabular}

Data are presented as number of patients (\%) unless otherwise indicated.

COVID-19, coronavirus disease 2019; GI, gastro-intestinal; TLC, total leukocytes count; CRP, C-reactive protein; AST, aspartate transaminase; ALT, alanine transaminase; ICU, Intensive Care Unit; NICU, Neonatal Intensive Care Unit.

\section{Laboratory parameters in COVID-19 patients}

Routine blood test reports of 3,231 COVID-19 cases were available from different sets of studies. The mean hemoglobin values were in the normal range in 3 analyzed studies (Table 3), which was probably due to the acute course of the illness. Predominantly, the cases had reduced total leukocyte count (TLC, 28.80\%) along with reduced platelet counts (27.69\%). Hemoglobin levels were not as acutely affected; however, thrombocytopenia was observed in approximately one-third of the patients. A minority of cases also presented with increased TLC (8.80\%). About $65 \%$ of cases had lymphocytopenia, as determined by a differential leukocyte count (DLC), which would be considered an initial response in viral illnesses. A few cases presented with increased neutrophil count (18.45\%), probably reflecting a component of superadded non-viral bacterial infection. The laboratory biochemical parameters (Table 3), such as albu$\min (53.72 \%)$ and lactate dehydrogenase (LDH, 45.71\%), were affected in all cases. The least affected parameter in our analysis was bilirubin. One third (34.84\%) of the cases presented with the raised natriuretic peptide responsible for natriuresis. There were no symptoms related to hypovolemic shock or increased frequency of urination. The impact of the raised natriuretic peptide could have been worse in cases 


\title{
Obstetrics \& Gynecology Science
}

\author{
Vol. 64, No. 2, 2021
}

with associated chronic renal failure. However, creatinine was elevated in $12.24 \%$ of cases. Other biochemical parameters, such as aspartate transaminase (AST) and alanine transaminase (ALT), were raised in (25.33\%) and $(21.86 \%)$, respectively. In the case of acute inflammatory cytokines (Table 3), C-reactive protein (CRP), which was raised among 59.51\% of total cases, followed by tumor necrosis factor (58.26\%), erythrocyte sedimentation rate (ESR, 57.14\%), interleukins (IL-2, 52.54\%; and IL-10, 30.0\%) were increased; however, prolactin level was increased in only $6.59 \%$ of the cases.

\section{COVID-19 profile associated with pregnancy}

A separate analysis of clinical symptoms and related laboratory parameters of COVID-19 infection among pregnant women was performed based on a published review article (Table 5). Here, representative studies from various regions were compiled. In these studies, fever (53.50\%) was again the most common complaint, followed by cough (35.41\%), malaise $(19.46 \%)$, shortness of breath $(13.58 \%)$, and diarrhea $(13.18 \%)$. Sore throat $(4.67 \%)$ was the least common symptom. Symptoms such as nausea, vomiting, and headache were not discussed in these studies, probably because they were overlooked by the patients themselves as these are common symptoms of pregnancy. In other biochemical investigations, there was a rise in CRP (50.73\%), followed by deranged values of liver enzymes such as AST (11.79\%) and ALT $(11.23 \%)$. The majority of pregnant women were treated with antibiotics (76.21\%), followed by antiviral (45.62\%) therapy, along with supportive management. A minority of the cases required intensive care admission (4.27\%) and invasive mechanical ventilation (1.79\%).

\section{Neonatal outcome in COVID-19 associated pregnancy}

In our analysis (Table 5), the gestational age ranged from 28 to 41 weeks. In total, 829 pregnancies were analyzed for gestational age, and 233 out of 829 (28.10\%) were preterm deliveries. A total of 691 cases were assessed for the mode of delivery, in which $553(77.07 \%)$ had a cesarean section, and 133 (21.93\%) had a spontaneous vaginal delivery. The neonatal outcome was satisfactory in terms of appearance, pulse, grimace, activity and respiration (APGAR) scores, which were ideal at 1 minute and 5 minutes after birth for most newborns. The mean birth weight was within the normal range; however, Smith et al. [28] reported 9/21 (42.86\%) cases with low birth weight irrespective of gestational age. Approximately 60/186 (32.25\%) cases were shifted to the Intensive Care Unit for a variety of reasons. The review collected information regarding the testing of COVID-19 infections among neonates. The samples utilized for testing included maternal blood, cord blood, amniotic fluid, placental tissue, and breast milk. We found that about 25/665 (3.75\%) neonates tested positive for COVID-19 infection after delivery, with the possibility of environmental exposure. In a report by Galang et al. [25], only 1 of $24(4.16 \%)$ amniotic fluid specimens tested positive, whereas cord blood $(0 / 12,0 \%)$, placental blood $(0 / 12,0 \%)$, and breast milk $(0 / 8,0 \%)$ specimens tested negative through PCR. In the study by Yoon et al. [26], 4 out of 201 neonates were infected by COVID-19 infection, which was detected in throat, nasal, and anal swabs. In a study by Trippella et al. [27], the overall analysis showed that out of a total of 248 neonates examined, approximately 16/248 (6.45\%) neonates were COVID-19 positive, and $93.55 \%$ neonates (born to COVID-19 positive mothers) were infection-free at the time of birth.

\section{Treatment and complications}

Management (Table 4) was predominantly symptomatic, with more than $50 \%$ of cases receiving a combination of interventions such as oxygen therapy (58.26\%), antiviral therapy (57.97\%), and antibiotics (50.35\%). Some patients received intravenous immunoglobulin $(22.71 \%)$ and systemic corticosteroids (33.15\%). A few cases had to undergo mechanical ventilation (13.60\%). Common complications observed in COVID-19 patients included acute cardiac injury (27.69\%) and acute respiratory distress syndrome (ARDS, 23.06\%), with only $1.6 \%$ developing disseminated intravascular coagulopathy (DIC). The recovery rate was approximately $41.89 \%$, with 1,013 cases out of a total of 2,418 cases discharged from the hospital after treatment. The mortality rate was approximately $6.56 \%$, with 172 deaths reported among 2,621 cases.

\section{Discussion}

The global society has experienced viral epidemics in the past, such as SARS in 2003, followed by MERS in 2012. SARS and MERS were public health calamities worldwide due to high death rates of $9.6 \%$ for SARS-CoV and $34.4 \%$ 


\section{Obstetrics \& Gynecology Science}

Harsh Goel, et al. Review of 3,000 COVID-19 cases

for MERS-CoV globally $[1,29,30]$. In the current scenario of an ongoing pandemic, the knowledge and experience of clinical management acquired in the past have been a great help in countries such as Taiwan, with only 7 deaths reported till date [30]. Many single-center-based experiences with relatively low numbers of cases have been published in the medical literature on COVID-19. The current review included the latest studies from January to May 2020 to analyze the clinical characteristics of COVID-19.

This review included more than 3000 patients and reflected the most recent data since the emergence of the novel coronavirus. The median age for symptomatic cases that required hospital care was 53 years (range, 47-62 years) (Table 1). Although the disease affected all age groups, the elderly patients had a relatively severe disease course. This analysis showed that a higher proportion of infected patients were male $(55.2 \%)$ compared with females (44.8\%). A similar finding was shown by a recently published study on 13,184 cases, where $55 \%$ of males were affected by COVID-19 infection [31]. The same study has also emphasized that cases with severe outcomes were predominantly males in the older age group. The predisposition for infection among males could have been attributed to a smoking habit, which is usually valid for most lung-related infective and neoplastic lesions. However, our limited data showed that approximately $86 \%$ of COVID-19 cases were non-smokers. This has also been discussed by Sanchez-Ramirez and Mackey [32]; they categorically identified a significant association between COVID-19 infection and a contemporary history of respiratory disease, current smoking, and former smoking.

Initial blood-based investigations (Table 3) showed no changes in the initial hemoglobin levels, and a predominance of reduced TLC counts among $27.69 \%$ of cases, along with reduced absolute lymphocyte counts among $65.08 \%$ of cases. This is a common laboratory finding associated with any viral illness. A similar finding of lymphopenia (72.3\%) and other deranged laboratory parameters was reported by Zhan et al. [33] in their cohort of 405 cases from China. A few cases $(18.45 \%)$ also had mildly raised neutrophils, probably representing a secondary bacterial infection, as viral illnesses make the epithelial mucosa more prone to bacterial infections. The most common symptoms (Table 1) were fever $(85.0 \%)$, cough $(70.63 \%)$, fatigue $(32.05 \%)$, chest tightness $(37.36 \%)$, dyspnea $(31.95 \%)$, shortness of breath $(31.19 \%)$, and expectoration (33.27\%). Fever and cough were also the most prevalent symptoms in other studies [33,34]. Minor symptoms included body myalgia (15.48\%), diarrhea $(11.39 \%)$, chills $(10.12 \%)$, and sore throat $(9.40 \%)$. Symptoms such as chest pain (5.54\%), abdominal pain (3.80\%), and hemoptysis (1.21\%) were rarely present. These rare symptoms always have the potential to masquerade as symptoms associated with angina, with cardiac pain as the elderly population is commonly being infected. These symptoms are primarily due to a generalized response against the infection, and the predisposition of the lungs to the infection makes cough and expectoration common symptoms.

COVID-19 was commonly associated with comorbidities such as hypertension $(21.60 \%)$, diabetes $(9.96 \%)$, and cardiovascular disease (5.78\%) (Table 2). The association of comorbidities with COVID-19 can be attributed to the general risk factor of increased infectivity among the elderly population. A recently published meta-analysis [35] of 25 studies involving 11 pre-existing comorbid conditions in 484 subjects showed that cardiovascular disease (risk ratio [RR] 2.25, 95\% confidence interval=1.60-3.17, number of studies $=14)$, hypertension (1.82 [1.43 to 2.32], $n=13$ ), diabetes (1.48 [1.02 to 2.15$], n=16)$ were among the top comorbid conditions significantly associated with COVID-19.

Analysis of the biochemical parameters revealed high levels of raised albumin $(53.72 \%), \mathrm{LDH}(45.71 \%)$, and natriuretic peptide (34.84\%), followed by various liver enzymes ALT (21.86\%) and AST (25.33\%) (Table 3). Parameters such as total bilirubin were raised among only $7.94 \%$ of the cases studied. LDH has been identified as a marker for severe prognosis in multiple diseases, including cancer and infection. In severe COVID-19 cases, the elevated LDH levels suggested that LDH may be correlated with lung damage and tissue injury [36-38]. This could indicate an overall compensatory response to the viral illness, which predominantly affects the respiratory system. Further, severe COVID-19 is associated with drastically elevated inflammatory cytokine levels, such as CRP (59.51\%) and tumor necrosis factor (TNF) (58.26\%). Biguenet et al. [39] confirmed that both LDH and CRP levels were elevated in the first ten days of the illness. In a few cases, ESR (57\%), IL-6 (54\%), IL- 2 (52\%), IL-10 (30\%), and IL-8 (10.16\%) increased with elevated prolactin levels (6.5\%). Although, there is no direct evidence of an association between COVID-19 and pro-inflammatory cytokines and chemokines in lung pathology, elevated serum cytokines signify a rapid multiplication of the virus and massive inflammatory 


\title{
Obstetrics \& Gynecology Science
}

\author{
Vol. 64, No. 2, 2021
}

infiltrates, resulting in acute lung injury $[40,41]$.

Symptomatic management was the mainstay of treatment, and more than $50 \%$ of cases required a combination of oxygen therapy (58.26\%), antiviral therapy (57.97\%), and antibiotics (50.35\%) (Table 4). Combinations of antiviral and antibiotic therapies were utilized to prevent secondary bacterial infections and further deterioration of the illness. The cases in our study had common complications related to cardiac injuries (27.69\%), followed by ARDS (23.06\%). A few cases of acute renal injury (6.76\%) and septic shock (4.15\%), and occasional cases of DIC (1.60\%) were also noted. In a study conducted in China, acute cardiac injury was the most common complication, with most cases dying between 2-19 days after admission to the ICU [42]. The treatment responses and associated comorbidities affected the duration of hospital stay and mortality. The overall mortality rate was about 172/2,621 (6.56\%), and the hospital recovery rate was approximately $41.89 \%$. The figures appear to represent the initial pandemic intervention in the most affected areas of the Asia Pacific region. However, in another study, a total of 17 deaths were reported from a meta-analysis of 12,258 cases in the United States of America [43]. This difference can be attributed to many factors such as clinical symptoms, smoking status, age, and other associated comorbid conditions. Other factors could be the ethnicity, time and sudden load of epidemic cases, available infrastructure, calamity preparedness, and so on. The countries that were not affected in the initial pandemic wave had some crucial period for preparedness and are expected to be relatively better in overall outcome figures.

Maternal and neonatal outcomes in COVID-19: Clinical and laboratory characteristics of COVID-19 among pregnant women were analyzed from 4 published retrospective studies (Table 5). The most common symptoms were fever (53.50\%) and cough (35.41\%), followed by malaise $(19.46 \%)$ and shortness of breath (13.58\%). The findings showed no difference in the frequency and characteristics of clinical symptoms between pregnant and non-pregnant women. Other published studies have reported similar findings $[44,45]$. Another published meta-analysis [46] of 28 studies on 11,432 pregnant women suggested that symptoms such as fever and myalgia were less frequently reported than in age-matched non-pregnant women, and pregnant women were more likely to need hospital admission. In addition to clinical features, other minor symptoms such as nausea and vomiting have been reported in non-pregnant women, but overlooked by pregnant women, probably because these symptoms are usually present throughout pregnancy. Laboratory parameters such as decreased TLC (80.20\%) and lymphocytes count (44.30\%), and elevated CRP levels (50.73\%), have also been reported in another study on 1,316 pregnant women, where lymphocytopenia and high CRP levels were observed in $55-100 \%$ of cases. The majority of the women were on antiviral and antibiotic therapy, along with oxygenation therapy support. In relatively later published studies [47], data related to hydroxyl-chloroquine (79.7\%) treatment have also been reported.

Most of the pregnancies had a gestational age of 28-41 weeks, and underwent an institutional cesarean section delivery $(77.07 \%)$. This is probably because most of the studies were from the referral hospital identified for COVID-19 management. A preterm delivery rate of $28.10 \%$ was observed in our study. A similar study from Ethiopia on pregnant women reported a preterm birth rate of $14.3 \%$ [46]. The findings related to APGAR score at 1 minute and 5 minutes after birth were within the normal range. The study also observed that the incidence of COVID-19 among newborn neonates was $25 / 665(3.75 \%)$. A total of 60/186 (32.25\%) neonates were transferred to neonatal intensive care. The odds of preterm birth were higher in COVID-19 infected pregnant women than in non-infected pregnant women [47]. Most studies $[44,45,48]$ have reported good APGAR scores along with events such as fetal distress, miscarriage, respiratory distress, and preterm delivery in pregnant women. COVID-19 infection was evaluated in maternal blood, cord blood, amniotic fluid, and breast milk; all of the studies $[43,45,48]$ reported no evidence of vertical transmission. The rate of infection of $3.1 \%$ among neonates in our study can probably be attributed to environmental exposure after birth. Overall, clinical and laboratory characteristics were similar in both pregnant and non-pregnant women.

The major limitation of this review is the geographic location; however, it could not be avoided as it coincided with the epicenter of COVID-19 infection. The selection of studies published in English also limits our review, as we may be missing some vital information published in the local languages. The selection bias for studies with a larger number of clinical and laboratory parameters might have resulted in the loss of vital information in smaller case series and reports. The pandemic is still ongoing; however, since it erupted in 


\section{Obstetrics \& Gynecology Science}

Harsh Goel, et al. Review of 3,000 COVID-19 cases

the Asia-Pacific region, the outcome will be based on regionspecific clinical and data management.

\section{Conclusion}

This review summarizes the clinical and laboratory parameters of COVID-19 patients, which would enable primary physicians and health care givers to provide an early and definitive diagnosis for an accelerated response. The primary objective for this in-depth analysis is to learn about common and less common signs and symptoms in normal as well as pregnant women. There is also the need to understand symptoms that might masquerade as pre-existing physiological conditions such as pregnancy or associated comorbidities. This may facilitate administrative policymakers and public health experts to deliver health policy-related decisions based on existing knowledge.

\section{Conflict of interest}

No potential conflict of interest relevant to this article was reported.

\section{Ethical approval}

The manuscript is based on the analysis of already published data. No human sample and confidential information was used. The manuscript is review article and do not require Ethics approval.

\section{Patient consent}

Written informed consent and the use of images from patients are not required for the publication.

\section{Funding information}

None.

\section{References}

1. Lu R, Zhao X, Li J, Niu P, Yang B, Wu H, et al. Genomic characterisation and epidemiology of 2019 novel coronavirus: implications for virus origins and receptor binding. Lancet 2020;395:565-74.

2. Zhou P, Yang XL, Wang XG, Hu B, Zhang L, Zhang W, et al. A pneumonia outbreak associated with a new coronavirus of probable bat origin. Nature 2020;579:270-3.

3. Huang C, Wang Y, Li X, Ren L, Zhao J, Hu Y, et al. Clinical features of patients infected with 2019 novel coronavirus in Wuhan, China. Lancet 2020;395:497-506.

4. Guan WJ, Ni ZY, Hu Y, Liang WH, Ou CQ, He JX, et al. Clinical characteristics of Coronavirus disease 2019 in China. N Engl J Med 2020;382:1708-20.

5. Wu Y, Ho W, Huang Y, Jin DY, Li S, Liu SL, et al. SARSCoV-2 is an appropriate name for the new coronavirus. Lancet 2020;395:949-50.

6. World Health Organization. WHO director-general's statement on IHR emergency committee on novel Coronavirus (2019-nCoV) [Internet]. Geneva: World Health Organization; c2020 [cited 2020 Jan 30]. Available from: https://www.who.int/dg/speeches/detail/who-directorgeneral-s-statement-on-ihr-emergency-committee-onnovel-coronavirus-(2019-ncov).

7. Coronaviridae Study Group of the International Committee on Taxonomy of Viruses. The species severe acute respiratory syndrome-related coronavirus: classifying 2019-nCoV and naming it SARS-CoV-2. Nat Microbiol 2020;5:536-44.

8. Ramadan N, Shaib H. Middle East respiratory syndrome coronavirus (MERS-CoV): a review. Germs 2019;9:35-42.

9. Zhu N, Zhang D, Wang W, Li X, Yang B, Song J, et al. A novel Coronavirus from patients with pneumonia in China, 2019. N Engl J Med 2020;382:727-33.

10. Lei $H$, Li Y, Xiao $S$, Lin CH, Norris SL, Wei $D$, et al. Routes of transmission of influenza A H1N1, SARS CoV, and norovirus in air cabin: comparative analyses. Indoor Air 2018;28:394-403.

11. Otter JA, Donskey C, Yezli S, Douthwaite S, Goldenberg SD, Weber DJ. Transmission of SARS and MERS coronaviruses and influenza virus in healthcare settings: the possible role of dry surface contamination. J Hosp Infect 2016;92:235-50.

12. Zumla A, Hui DS, Perlman S. Middle East respiratory syn- 


\section{Obstetrics \& Gynecology Science}

Vol. 64, No. 2, 2021

drome. Lancet 2015;386:995-1007.

13. Minodier L, Charrel RN, Ceccaldi PE, van der Werf S, Blanchon T, Hanslik T, et al. Prevalence of gastrointestinal symptoms in patients with influenza, clinical significance, and pathophysiology of human influenza viruses in faecal samples: what do we know? Virol J 2015;12:215.

14. Li Q, Guan X, Wu P, Wang X, Zhou L, Tong $Y$, et al. Early transmission dynamics in Wuhan, China, of novel Coronavirus-infected pneumonia. N Engl J Med 2020;382:1199-207.

15. Chen N, Zhou M, Dong X, Qu J, Gong F, Han Y, et al. Epidemiological and clinical characteristics of 99 cases of 2019 novel coronavirus pneumonia in Wuhan, China: a descriptive study. Lancet 2020;395:507-13.

16. Kim KH, Tandi TE, Choi JW, Moon JM, Kim MS. Middle East respiratory syndrome coronavirus (MERS-CoV) outbreak in South Korea, 2015: epidemiology, characteristics and public health implications. J Hosp Infect 2017:95:207-13.

17. World Health Organization. WHO COVID-19: case definitions updated in public health surveillance for COVID-19 [Internet]. Geneva: World Health Organization; c2020 [cited 2020 Jan 30]. Available from: https://www. who.int/publications-detail-redirect/WHO-2019-nCoVSurveillance_Case_Definition-2020.1

18. Feng Y, Ling Y, Bai T, Xie Y, Huang J, Li J, et al. COVID-19 with different severities: a multicenter study of clinical features. Am J Respir Crit Care Med 2020;201:1380-8.

19. Yu X, Sun X, Cui P, Pan H, Lin S, Han R, et al. Epidemiological and clinical characteristics of 333 confirmed cases with coronavirus disease 2019 in Shanghai, China. Transbound Emerg Dis 2020;67:1697-707.

20. Chen T, Wu D, Chen H, Yan W, Yang D, Chen G, et al. Clinical characteristics of 113 deceased patients with coronavirus disease 2019: retrospective study. BMJ 2020;368:m1091.

21. Chen T, Dai Z, Mo P, Li X, Ma Z, Song S, et al. Clinical characteristics and outcomes of older patients with coronavirus disease 2019 (COVID-19) in Wuhan, China: a single-centered, retrospective study. Gerontol A Biol Sci Med Sci 2020;75:1788-95.

22. Cai Q, Huang D, Ou P, Yu H, Zhu Z, Xia Z, et al. COVID-19 in a designated infectious diseases hospital outside Hubei Province, China. Allergy 2020;75:1742-52.
23. Li X, Xu S, Yu M, Wang K, Tao Y, Zhou Y, et al. Risk factors for severity and mortality in adult COVID-19 inpatients in Wuhan. J Allergy Clin Immunol 2020;146:110-8.

24. Juan J, Gil MM, Rong Z, Zhang Y, Yang H, Poon LC. Effect of coronavirus disease 2019 (COVID-19) on maternal, perinatal and neonatal outcome: systematic review. Ultrasound Obstet Gynecol 2020;56:15-27.

25. Galang RR, Chang K, Strid P, Snead MC, Woodworth $K R$, House LD, et al. Severe Coronavirus infections in pregnancy: a systematic review. Obstet Gynecol 2020;136:262-72.

26. Yoon SH, Kang JM, Ahn JG. Clinical outcomes of 201 neonates born to mothers with COVID-19: a systematic review. Eur Rev Med Pharmacol Sci 2020;24:7804-15.

27. Trippella G, Ciarcià M, Ferrari M, Buzzatti C, Maccora I, Azzari $C$, et al. COVID-19 in pregnant women and neonates: a systematic review of the literature with quality assessment of the studies. Pathogens 2020;9:485.

28. Smith V, Seo D, Warty R, Payne O, Salih M, Chin KL, et al. Maternal and neonatal outcomes associated with COVID-19 infection: a systematic review. PLoS One 2020;15:e0234187.

29. World Health Organization. Summary of probable SARS cases with onset of illness from 1 November 2002 to 31 July 2003 [Internet]. Geneva: World Health Organization; c2020 [cited 2020 Feb 10]. Available from: https:// www.who.int/csr/sars/country/table2004_04_21/en/.

30. World Health Organization. Middle East respiratory syndrome coronavirus (MERS-CoV) [Internet]. Geneva: World Health Organization; c2020 [cited 2020 Feb 10]. Available from: http://www.who.int/emergencies/merscov/en/.

31. Taiwan center for disease control. Weekly number of suspected COVID-19 cases. [Internet]. Taipei city: Taiwan center for disease control; c2020 [cited 2020 Sep 13]. Available from: https://www.cdc.gov.tw/En.

32. Sanchez-Ramirez DC, Mackey D. Underlying respiratory diseases, specifically COPD, and smoking are associated with severe COVID-19 outcomes: a systematic review and meta-analysis. Respir Med 2020;171:106096.

33. Zhan T, Liu M, Tang Y, Han Z, Cheng X, Deng J, et al. Retrospective analysis of clinical characteristics of 405 patients with COVID-19. J Int Med Res 2020 Aug 31 [Epub]. https://doi.org/10.1177/0300060520949039.

34. Xiao M, Hou M, Liu X, Li Z, Zhao Q. Clinical characteris- 


\section{Obstetrics \& Gynecology Science}

Harsh Goel, et al. Review of 3,000 COVID-19 cases

tics of 71 patients with coronavirus disease 2019. Zhong Nan Da Xue Xue Bao Yi Xue Ban 2020;45:790-6.

35. Ssentongo P, Ssentongo AE, Heilbrunn ES, Ba DM, Chinchilli VM. Association of cardiovascular disease and 10 other pre-existing comorbidities with COVID-19 mortality: a systematic review and meta-analysis. PLoS One 2020;15:e0238215.

36. Wu C, Chen X, Cai Y, Xia J, Zhou X, Xu S, et al. Risk factors associated with acute respiratory distress syndrome and death in patients with Coronavirus disease 2019 pneumonia in Wuhan, China. JAMA Intern Med 2020;180:934-43.

37. Zhou F, Yu T, Du R, Fan G, Liu Y, Liu Z, et al. Clinical course and risk factors for mortality of adult in patients with COVID-19 in Wuhan, China: a retrospective cohort study. Lancet 2020;395:1054-62.

38. Yang $X, Y u ~ Y, X u J$, Shu $H$, Xia J, Liu H, et al. Clinical course and outcomes of critically ill patients with SARSCoV-2 pneumonia in Wuhan, China: a single-centered, retrospective, observational study. Lancet Respir Med 2020;8:475-81.

39. Biguenet A, Bouiller K, Marty-Quinternet S, Brunel AS, Chirouze C, Lepiller Q. SARS-CoV-2 respiratory viral loads and association with clinical and biological features. J Med Virol 2020 Sep 5 [Epub]. https://doi. org/10.1002/jmv.26489.

40. Min CK, Cheon S, Ha NY, Sohn KM, Kim Y, Aigerim A, et al. Comparative and kinetic analysis of viral shedding and immunological responses in MERS patients representing a broad spectrum of disease severity. Sci Rep 2016;6:25359.

41. Channappanavar R, Perlman S. Pathogenic human coronavirus infections: causes and consequences of cytokine storm and immunopathology. Semin Immunopathol 2017;39:529-39.

42. Lu J, Zhang Y, Cheng G, He J, Wu F, Hu H, et al. Clinical characteristics and outcomes of adult critically ill patients with COVID-19 in Honghu, Hubei Province. Nan Fang Yi Ke Da Xue Xue Bao 2020;40:778-85.

43. Patel U, Malik P, Usman MS, Mehta D, Sharma A, Malik FA, et al. Age-adjusted risk factors associated with mortality and mechanical ventilation utilization amongst COVID-19 hospitalizations-a systematic review and meta-analysis. SN Compr Clin Med 2020 Aug 29 [Epub]. https://doi.org/10.1007/s42399-020-00476-w.

44. Harapan $H$, Itoh N, Yufika A, Winardi W, Keam S, Te H, et al. Coronavirus disease 2019 (COVID- 19): a literature review. J Infect Public Health 2020;13:667-73.

45. Chen H, Guo J, Wang C, Luo F, Yu X, Zhang W, et al. Clinical characteristics and intrauterine vertical transmission potential of COVID-19 infection in nine pregnant women: a retrospective review of medical records. Lancet 2020;395:809-15.

46. Allotey J, Stallings E, Bonet M, Yap M, Chatterjee S, Kew $T$, et al. Clinical manifestations, risk factors, and maternal and perinatal outcomes of coronavirus disease 2019 in pregnancy: living systematic review and meta-analysis. BMJ 2020;370:m3320.

47. Diriba K, Awulachew E, Getu E. The effect of coronavirus infection (SARS-CoV-2, MERS-COV, and SARS-CoV) during pregnancy and the possibility of vertical maternal-fetal transmission: a systematic review and metaanalysis. Eur J Med Res 2020;25:39.

48. Panahi L, Amiri M, Pouy S. Risks of novel coronavirus disease (COVID-19) in pregnancy; a narrative review. Arch Acad Emerg Med 2020;8:e34. 